\title{
Utilising one minute and four minute recovery when employing the resistance training contrast method does not negatively affect subsequent jump performance in the presence of concurrent training .
}

\author{
Dean Ritchie ${ }^{\text {Corresp., 1, } 2}$, Justin Keogh ${ }^{1,3,4,5}$, Peter Reaburn ${ }^{1}$, Jonathan D Bartlett ${ }^{1,6}$ \\ 1 Faculty of Health Science and Medicine, Bond University, Gold Coast, Queensland, Australia \\ 2 Gold Coast Suns FC, Gold Coast, Queensland, Australia \\ 3 Faculty of Science, Health, Education and Engineering, University of the Sunshine Coast, Sunshine Coast, Queensland, Australia \\ 4 Kasturba Medical College, Manipal Academy of Higher Education, Karnataka, India \\ ${ }^{5}$ Sports Performance Research Centre New Zealand, Auckland University of Technology, Auckland, New Zealand \\ 6 Institute for Health and Sport, Victoria University, Melbourne, VIC, Australia \\ Corresponding Author: Dean Ritchie \\ Email address: dean.ritchie@goldcoastfc.com.au
}

Background: Little is known about contrast training and post-activation performance enhancement (PAPE) in a same day concurrent training model. The aim of the current study was to examine the use of two short duration (1-min and 4-min) recovery periods on drop jump performance in same day concurrently trained athletes.

Methods: Ten professional Australian Rules footballers (age, $20.6 \pm 1.9 \mathrm{yr}$; height, $184.8 \pm 6.9 \mathrm{~cm}$; body mass, $85.8 \pm 8.4 \mathrm{~kg}$ ) completed two resistance training sessions with different PAPE recovery durations; 1-min and 4-min, $1 \mathrm{~h}$ following a field-based endurance session. Baseline (pre) drop jumps were compared to post-test maximal drop jumps, performed after each set of 3 squats (where each participant was encouraged to lift as heavy as they could), to determine changes between 1-min and 4-min recovery periods. Data were analyzed by fitting a mixed model (significance was set at $P \leq 0.05$ ). Corrected Hedges' g standardised effect sizes $\pm 95 \%$ confidence limits were calculated using group means \pm SDs.

Results: There were no significant differences between baseline and experimental sets 1, 2 and 3 for reactive strength index (RSI), flight time, and total and relative impulse for either recovery duration. However, for contact time, 1-min baseline was significantly different from set 2 (mean difference; $95 \% \mathrm{Cl}$, $0.029 ; 0.000$ to $0.057 \mathrm{~s}, \mathrm{P}=0.047, \mathrm{ES} ; 95 \% \mathrm{Cl},-0.27 ;-1.20$ to 0.66 ). For RSI and flight time, 1-min was significantly higher than 4-min (RSI: $0.367 ; 0.091$ to $0.642, \mathrm{P}=0.010, \mathrm{ES} ; 95 \% \mathrm{Cl}, 0.52 ;-0.37$ to 1.42 ; flight time: $0.033 ; 0.003$ to $0.063 \mathrm{~s}, \mathrm{P}=0.027, \mathrm{ES} ; 95 \% \mathrm{Cl}, 0.86 ;-0.06$ to 1.78 ). '

Discussion: Short recovery periods of 1-min may be a time-efficient form of prescribing strength-power exercise in contrast loading schemes. Longer recovery periods do not appear to benefit immediate, subsequent performance. 
1 Manuscript Title: Utilising one minute and four minute recovery when employing the resistance

2 training contrast method does not negatively affect subsequent jump performance in the presence

3 of concurrent training.

4

5 Running Head: Post-activation performance enhancement in team sports athletes

6 Research was conducted at Metricon Stadium, the training facility of the Gold Coast Suns

7 Football Club.

8 Authors: Dean Ritchie ${ }^{1,2}$, Justin Keogh ${ }^{1,3,4,5}$, Peter Reaburn ${ }^{1}$ and Jonathan D. Bartlett ${ }^{1,6}$.

10 Affiliations:

$11{ }^{1}$ Faculty of health science and medicine, Bond University, Gold Coast, Queensland, Australia

$12{ }^{2}$ Gold Coast Suns FC, Gold Coast, Queensland, Australia

$13{ }^{3}$ Sports Performance Research Centre New Zealand, AUT University, Auckland, New Zealand

$14{ }^{4}$ Faculty of science, Health, Education and Engineering, University of the Sunshine Coast,

15 Sunshine Coast, Queensland, Australia

$16{ }^{5}$ Kasturba Medical College, Manipal Academy of Higher Education, Karnataka, India

$17{ }^{6}$ Institute for Health and Sport, Victoria University, Melbourne, Victoria, Australia

18

19

20

21

22

23
Corresponding Author:

Dean Ritchie

Bond Institute of Health and Sport, Faculty of Health Sciences and Medicine,

Bond University

Gold Coast,

Bond University,

Queensland

Australia, 8001

Email: Dean.Ritchie@GoldCoastFC.com.au

Tel: +61 408941313 
Background: Little is known about contrast training and post-activation performance enhancement (PAPE) in a same day concurrent training model. The aim of the current study was to examine the use of two short duration (1-min and 4-min) recovery periods on drop jump performance in same day concurrently trained athletes.

Methods: Ten professional Australian Rules footballers (age, $20.6 \pm 1.9$ yr; height, $184.8 \pm 6.9$ $\mathrm{cm}$; body mass, $85.8 \pm 8.4 \mathrm{~kg}$ ) completed two resistance training sessions with different PAPE recovery durations; 1-min and 4-min, $1 \mathrm{~h}$ following a field-based endurance session. Baseline (pre) drop jumps were compared to post-test maximal drop jumps, performed after each set of 3 squats (where each participant was encouraged to lift as heavy as they could), to determine changes between 1-min and 4-min recovery periods. Data were analyzed by fitting a mixed model (significance was set at $\mathrm{P} \leq 0.05$ ). Corrected Hedges' g standardised effect sizes $\pm 95 \%$ confidence limits were calculated using group means \pm SDs

Results: There were no significant differences between baseline and experimental sets 1, 2 and 3 for reactive strength index (RSI), flight time, and total and relative impulse for either recovery duration. However, for contact time, 1-min baseline was significantly different from set 2 (mean difference; $95 \% \mathrm{CI}, 0.029 ; 0.000$ to $0.057 \mathrm{~s}, \mathrm{P}=0.047$, ES; $95 \% \mathrm{CI},-0.27 ;-1.20$ to 0.66 ). For RSI and flight time, 1-min was significantly higher than 4-min (RSI: $0.367 ; 0.091$ to $0.642, \mathrm{P}=$ 0.010, ES; $95 \%$ CI, $0.52 ;-0.37$ to 1.42 ; flight time: $0.033 ; 0.003$ to $0.063 \mathrm{~s}, \mathrm{P}=0.027, \mathrm{ES} ; 95 \%$ CI, $0.86 ;-0.06$ to 1.78$)$.

Discussion: Short recovery periods of 1-min may be a time-efficient form of prescribing strength-power exercise in contrast loading schemes. Longer recovery periods do not appear to 52 benefit immediate, subsequent performance. 
53 Key Words: Training organisation, post-activation potentiation, periodisation, team sport. 
54

55

56

57

58

59

60

61

62

63

64

65

66

67

68

69

70

71

72

73 The benefit of using the contrast training method reflects the ability to utilise post-activation

74

75

76

\section{INTRODUCTION}

Within the strength and conditioning literature, two commonly used models exist to periodise within-session resistance training; the traditional and contrast training methods. The traditional training approach structures training in accordance to the estimated cost of fatigue whereby plyometric, power and compound exercises are completed at the beginning of the resistance training session $(1,2)$. The second method is contrast training which typically involves the performance of 3-5 repetitions with high loads of compound exercises, e.g., squats or power cleans, shortly followed by a lighter power or plyometric exercise (3). This contrast mode of resistance training has become popular within team sport settings (4-6).

In the context of high-performance team sports, drop jumps are commonly utilised when executing a number of key technical skills that directly contribute to sport performance. While jump height has typically been the primary outcome assessed in jumping studies, ground contact time may also be important, as the same jump height achieved by a shorter ground contact time leads to a more efficient jump strategy. Reactive strength index (RSI) takes into account both jump height and ground contact time from drop jumps and is calculated as jump height divided by ground contact time (7). Consequently, any contrast training related chronic improvements in an athlete's RSI and thus drop jump ability may improve the performance of many key technical skills and competitive match play.

potentiation (PAP) across multiple training sessions, thereby increasing the potential for positive adaptations in the long-term. PAP is the acute enhancement of force or muscle twitch contraction after a previous conditioning contraction or maximal voluntary contraction (8-10). Whilst PAP is 
77 typically associated with enhancements in muscle twitch properties, recent research indicates that

78

post-activation performance enhancement (PAPE) is a more appropriate term in relation to contrast training as it reflects the ability to enhance voluntary force production (11).

PAPE is utilised on the premise that the preceding strength-based movement may result in a complementary improvement in performance of the following jump or plyometric movement $(10,12)$. PAPE is generally elicited when a small number of dynamic repetitions $(\leq 5)$ are performed as explosively as possible with loads of $80-100 \%$ of repetition maximum $(9,10)$. Whilst the mechanistic underpinnings of PAP reflect two major pathways; the phosphorylation of myosin regulatory light chains $(10,13-16)$ and alterations in neural stimulation (10), PAPE mechanisms are more related to changes in muscle temperature and alterations to muscle force; effects purported to be driven by fluid changes to the working musculature (11).

Of specific relevance to the planning, prescription and organisation of resistance training in team sports, the rest interval between the conditioning contraction and subsequent plyometric or power exercise has been previously reported to affect the PAPE effect (12). A meta-analysis has indicated that 8 to 12 minutes of recovery after the conditioning contraction is required to induce the greatest PAPE response (12). However, in the context of team sport training, where large numbers of athletes train simultaneously within scheduled time blocks, the elongated recovery period of 8-12 minutes is impractical. In team sport athletes, a positive PAPE response has been demonstrated when utilising short ( $\leq 4$ minute) recovery periods. For example, Mitchell and Sale (17) demonstrated in resistance-trained university rugby union players a $2.9 \%$ increase in CMJ height 4 minutes after 5-RM back squat with a self-selected load. The variations in the optimal 
100 recovery period to maximise the PAPE response may reflect variations in the PAPE protocol

101 (e.g. intensity of the conditioning stimulus and the magnitude and time course of resulting

102 fatigue) and the characteristics (e.g. strength and fatigability) of the participants (12, 18). This

103 highlights the reciprocal relationship between PAPE and fatigue as evidenced by varying results

104 in performance outcomes (18). The PAPE recovery period presents as a challenge, particularly

105 in-season, since limited time is allocated to resistance-training $(19,20)$. Thus, a shorter recovery

106 duration between contraction stimulus and subsequent plyometric/jump exercise is highly

107 appealing as it represents a more ecologically valid training methodology for use in high-

108 performance sport.

110 In further considering the ecological relevance of PAPE duration time and its increasing

111 utilisation within team sport settings, no previous PAPE related research has been conducted in

112 high level athletes performing same day concurrent training $(\mathrm{CT})(4,6)$. Indeed, given that

113 concurrently-trained athletes typically, by way of their training history, have high strength levels

114 and are generally fatigue-resistant $(20,21)$, they may be able to demonstrate significant PAPE

115 effects with shorter rest periods than that commonly recommended in the strength and

116 conditioning literature $(12,17)$. Moreover, the training phase (i.e., pre- versus in-season) (20)

117 and distribution of load (22) across a week are altered highlighting why PAPE recovery time

118 course may be important in relation to resistance training organisation. Accordingly, the aim of

119 the current study was to examine the effect of short duration contrast training recovery time

120 course (1-min) versus longer duration recovery time course (4-min) on drop jump (DJ)

121 performance in the presence of same day concurrent training. The authors hypothesised that both 
122 short duration protocols would not attenuate subsequent DJ perfromance and will provide an

123 ecologically valid recovery time course for PAPE in same day CT athletes.

\section{MATERIALS AND METHODS}

125 In a randomised crossover design, participants completed two resistance training (RT) sessions

126 with different PAPE recovery durations; 1-min and 4-min, following a field-based endurance

127 session (endurance session external load matched between 1-min and 4-min protocols). Briefly,

128 participants undertook a standardised same-day CT protocol where endurance training

129 (predominately skill-based drills with the addition of top-up running) was completed in the

130 morning followed by a RT session in the afternoon (Fig. 1) (23). All participants were provided 1

131 hour of recovery between the endurance and RT training sessions and 48 hours was allocated

132 between the contrast training testing session and previous training session. Three baseline drop

133 jumps (DJ) were performed after a standardised warm-up, followed by 3 sets of 3 squats where

134 each participant was encouraged to lift as heavy as possible. DJ were performed with either 1-

135 min or 4-min recovery after each set of squats. All participants had previously participated in and

136 were familiar with heavy barbell box squatting and plyometrics movements where a range of

137 force plate measured training metrics were assessed.

138

139 Ten professional Australian rules football (AF) athletes (mean \pm SD: age, $20.6 \pm 1.9 \mathrm{yr}$; height,

$140184.8 \pm 6.9 \mathrm{~cm}$; body mass, $85.8 \pm 8.4 \mathrm{~kg}$; Box Squat 1RM, $150 \pm 16 \mathrm{~kg}$; elite training age, $3.0 \pm$

$1411.2 \mathrm{yr}$ ) from the same Australian Football League (AFL) club participated in this in-season study.

142 The participants competed in the national AFL competition with each providing written informed

143 consent. All participants were required to be free from any injury or medical conditions

144 throughout the data collection period that may affect their performance or endanger their health. 
145 If players suffered an injury, defined as pain resulting in modified load, data was excluded from

146 final analysis. The project was approved by the University's Human Research Ethics Committee

147 (DR03167).

149 Participants arrived at the training facility between 7:00 and 8:00 am before completing 150 individual preparation for a team-based skills/endurance session. External training load of the 151 field-based outdoor sessions (field-based skills/endurance) was monitored via Catapult S5 152 OptimEye (Catapult Innovations, Docklands Vic, Australia) global position system (GPS). 153 Training metrics obtained were Total Distance meters (m) (TD), Total High Speed Running (>15 $\left.154 \mathrm{~km} \cdot \mathrm{h}^{-1}\right)(\mathrm{m})(\mathrm{HSR})$, Total Distance above $75 \%$ of an individual's maximum velocity (m) (75\%), 155 Total Distance above $85 \%$ of an individual's maximum velocity $(\mathrm{m})(85 \%)$ and mean running 156 speed $\left(\mathrm{m} \cdot \mathrm{min}^{-1}\right)$. Maximal velocities were obtained as previously described (24). Each player

157 wore the same GPS device across both outdoor sessions to account for between unit errors (25) 158 as per manufacturer instructions (20). The accuracy and reliability of $10 \mathrm{~Hz}$ GPS units for 159 quantifying the movement demands of team sport athletes have been previously reported (26).

After the completion of the field-based skills/endurance session, participants consumed a mixed meal containing variable amounts of carbohydrate and protein, targeted towards each participants' own personal body composition and training goals. This was followed by passive

164 rest until commencement of the RT session. At the beginning of the subsequent RT session, 165 participants completed a standardised warm up which included bodyweight squats, mini-band 166 lateral walks and pogo jumps followed by 3 submaximal DJ's. The participants were then given

1672 minutes recovery before their baseline DJ were recorded. Participants were instructed to place 
168 hands on hips and to hold them there throughout the jump. Participants were then instructed to 169 step off the box $(30 \mathrm{~cm})$ and land with two feet on a force place (400 series force plate, Fitness

170 Technology, Adelaide, SA, Australia) simultaneously ensuring a short ground contact time and

171 maximal rebound jump height with the aim to jump as high as possible. No knee bend was

172 allowed during flight phase. If knee bend was observed, the jump was repeated. This type of DJ

173 has previously been referred to as a bounce drop jump (7). After 3 baseline jumps were

174 completed, 2 minutes of recovery time was allowed then participants commenced the contrast

175 training protocol. Table 1 displays the Intraclass correlation coefficients (ICC) and coefficient of

176 variation $(\mathrm{CV})$ for baseline DJ outcome measures.

177 Table 1 about here

178

179 Participants were instructed to complete a Barbell box squat utilising a self-selected 'heavy' load 180 with participants instructed to lift as much as possible for the prescribed sets and reps. Three 181 repetitions of the Barbell box squat $(74 \% \pm 9 \%$ of $1-\mathrm{RM})$ followed by a 1 -min or 4 -min rest 182 period between each squat set before performing 3 maximal DJ repetitions. Force plate variables 183 of contact time (CT) (s), flight time (FT) (s), impulse (N.s), relative impulse (N.s.kg-1) and

184

reactive strength index $(\mathrm{RSI})(\mathrm{RSI}=$ flight time $(\mathrm{s}) /$ contact time $(\mathrm{s}))(27,28)$ were collected and recorded via the associated computer software (Ballistic Measurement System; Fitness

Technology). These methods have been previously proven as reliable and valid measures of assessing changes in lower body power (29-32).

Prior to the analysis of outcome measures, Shapiro-Wilk tests for normality and lognormality were demonstrated for the preceding sport specific running loads and PAPE squat conditioning 
191 stimulus. All outcome measures for DJ performance including modified RSI, impulse, relative

192 impulse, flight time and contact time are presented as mean \pm standard deviation. Paired t-test

193 and Wilcoxon tests were utilized to assess significant differences between individual GPS

194 training metrics (total distance, high-speed running, distance covered $>75 \%$ and $85 \%$ maximal

195 velocity and $\mathrm{m} / \mathrm{min}$ ) and PAPE squat tonnage between the 1-min and 4-min protocols. The

196 subsequent analyses were carried out separately for each dependent variable (RSI, impulse,

197 relative impulse, flight time and contact time) with 3 comparisons per family (sets). Due to

198 technical (a malfunction of the force plate software) constraints there were 10 missing values

199 across the study of random participants. Therefore, data were analyzed by fitting a mixed model,

200 rather than by repeated measures ANOVA. Significance was set at $\mathrm{P} \leq 0.05$. All data were

201 analyzed using (GraphPad Prism Version 8.04.1, GraphPad Software, La Jolla, CA).

202 Subsequently, bias corrected Hedges' g standardised effect sizes $\pm 95 \%$ confidence limits were

203 calculated using group means \pm SDs via a customised Excel spreadsheet. Modified Cohens' d

204 thresholds of small (0.2), moderate $(0.5)$, and large $(>0.8)$ were used to determine the magnitude 205 of effect (33).

206

207 Figure 1 about here

Preceding field-based skills/endurance (sport specific) same day running loads that precede the

PAPE protocols are displayed in Table 2 with PAPE squat conditioning stimulus tonnage shown

211 in Table 3. There were no significant differences in either sport-specific running loads or PAPE conditioning stimulus load between the 1-min and 4-min protocols. 
214 Table 2 about here

215 Table 3 about here

216

217 There were no significant difference between baseline 1-min and 4-min conditions for any of the

218 jump performance variables (Fig. 2). Within condition analyses between baseline and

219 experimental sets 1, 2 and 3 revealed no significant difference for RSI, flight time, total and

220 relative impulse (Fig. 3 and Fig. 4). However, for contact time, 1-min baseline was different

221 from set 2 only (mean difference; $95 \%$ CI, $0.029 ; 0.000$ to 0.057 s, P $=0.047, \mathrm{ES} ; 95 \%$ CI, -0.27 ;

$222-1.20$ to 0.66 ) with no significant differences observed for 4-min.

223

224 Figure 2 about here

225 Figure 3 about here

226 Figure 4 about here

227

228 Between condition set comparisons for both conditions are summarized in Fig. 5. For RSI, there

229 was no difference between conditions for set 1 (mean difference; 95\% CI, 0.200; -0.214 to 0.612 , $230 \mathrm{P}=0.472, \mathrm{ES} ; 95 \% \mathrm{CI}, 0.26 ;-0.62$ to 1.14$)$ and set $2(0.309 ;-0.114$ to $0.733, \mathrm{P}=0.162, \mathrm{ES}$;

$23195 \%$ CI, 0.45; -0.48 to 1.39). However, for set 3, RSI was significantly higher for the 1-min than

232 4-min condition $(0.367 ; 0.091$ to $0.642, \mathrm{P}=0.010, \mathrm{ES} ; 95 \% \mathrm{CI}, 0.52 ;-0.37$ to 1.42$)$ (Fig. 5a). 233

234 Figure 5 about here 235 
236 Consistent with RSI, there was no difference between conditions for flight time for set $1(0.027$; -

2370.026 to $0.080 \mathrm{~s}, \mathrm{P}=0.435$, ES; $95 \% \mathrm{CI}, 0.62 ;-0.28$ to 1.52$)$ and $\operatorname{set} 2(0.014 ;-0.048$ to $0.078 \mathrm{~s}$, $238 \mathrm{P}=0.870, \mathrm{ES} ; 95 \% \mathrm{CI}, 0.34 ;-0.59$ to 1.27$)$. However, for set 3 , flight time was greater for the 1239 min than 4-min condition $(0.033 ; 0.003$ to $0.063 \mathrm{~s}, \mathrm{P}=0.027, \mathrm{ES} ; 95 \% \mathrm{CI}, 0.86 ;-0.06$ to 1.78$)$ 240 (Fig. 5d).

242 For absolute impulse (N.s), there was no difference between conditions for set $1(373 ;-2932$ to 3678 N.s, $\mathrm{P}=0.984$, ES; $95 \%$ CI, $0.07 ;-0.81$ to 0.94$)$, set 2 (2348; -1763 to 6459 N.s, $\mathrm{P}=0.314$, ES; $95 \%$ CI, $0.37 ;-0.56$ to 1.30$)$ or set $3(2744 ;-255$ to 5744 N.s, P = 0.074, ES; $95 \%$ CI, 0.50; conditions (Set 1: 6.54; -31.47 to 44.56 N.s. $\mathrm{kg}^{-1}, \mathrm{P}=0.948$, ES; 95\% CI, 0.08; -0.79 to 0.96; Set 2: $34.57 ;-17.72$ to 86.86 N.s.kg-1, $\mathrm{P}=0.217$, ES; $95 \% \mathrm{CI}, 0.42 ;-0.51$ to 1.36 ; Set $3: 33.92 ;-5.58$ to 73.42 N.s.kg-1, $\mathrm{P}=0.097, \mathrm{ES} ; 95 \% \mathrm{CI}, 0.45 ;-0.44$ to 1.33 ) (Fig. 5c).

For contact time there was no difference between conditions for any of the 3 sets (Set 1: 0.003; -

0.034 to $0.040 \mathrm{~s}, \mathrm{P}=0.992, \mathrm{ES} ; 95 \% \mathrm{CI}, 0.06 ;-0.82$ to 0.94 ; Set $2:-0.014 ;-0.053$ to $0.024 \mathrm{~s}, \mathrm{P}=$

0.622, -ES; $95 \%$ CI, $0.27 ;-1.20$ to 0.66 ; Set $3:-0.017 ;-0.038$ to 0.004 s, P $=0.138, \mathrm{ES} ; 95 \% \mathrm{CI}$, $-0.26 ;-1.14$ to 0.62$)$ (Fig. 5e).

254

257 The major finding of the current study was that 1-min and 4-min had no negative effect on 258 subsequent drop jump performance compared to respective baselines following same day skills- 
259 specific endurance training. Furthermore, when comparing 1-min versus 4-min between sets,

260 both RSI and flight time significantly improved in set 3 with 1-min compared to 4-min protocol.

261 Taken together, these data have implications for the organization and planning of contrast

262 resistance training in team sport settings where many athletes have relatively limited time to

263 perform resistance training. Specifically, the results suggest that concurrent trained athletes with

264 a strong aerobic/anaerobic conditioning base may be able to utilize shorter RT rest periods than

265 is currently recommended for most other individuals.

266

267 The benefit of using CT reflects the ability to utilise preceding strength-based movement to

268 improve performance of the subsequent jump or plyometric movement (10). In the current study

269 no detrimental effect of utilising short 1- and 4-min recovery durations was observed on

270 subsequent drop jump performance following field-based skills/endurance running loads. This

271 finding contributes to the current contrast training research literature, where a previous meta-

272 analysis suggest that 8-12 minutes recovery after the conditioning contraction produces the

273 greatest PAPE effect (12). Importantly, individual studies presented in the meta-analysis indicate

274 that the resultant PAPE may be observed anywhere from between 4-24 minutes (12). However,

275 due to the complex nature of team sports weekly dense schedules (20) where several other

276 aspects of coaching, recovery and mental well-being are required, if employing the

277 recommended 8-12 minute recovery period (12) between 3-4 sets of heavy back squat exercise

278 and subsequent plyometric exercise, the time to complete these exercises would be $>40$ minutes.

279 This length of recovery period provides limited time to complete supplementary exercises within

280 a typical resistance training session of $60 \mathrm{~min}$ and is impractical in the applied high-performance

281 team training setting. 
283 The utility of the DJ in the current study and not countermovement jump or squat jump can be

284

285

286

287

288

289

290

291

292

293

294

295

296

297

298

299

300

301

302

303

304

explained by the importance of high levels of reactive strength produced over short contact times required as part of an efficient $\mathrm{DJ}(4,5,34)$, and the direct relevance of the vertical jump to Australian Rules football specific actions. The short contact is represented by a fast stretch shortening cycle (SSC) which is defined as ground reaction forces ranging from 100-250 ms (35). The present results showed an increase in RSI and FT in set 3 of 1-min compared to 4-min. These findings are important as RSI, expressed as the relationship between flight and contact time, represents explosive strength and the ability to develop maximal force in minimal time. Indeed, the ability to perform explosive actions utilising the SSC is a requisite for most sports (36). Given that high-intensity intermittent activity requires accelerations, change of direction and decelerations to be performed with maximal force almost instantaneously through competition $(20,26)$, RSI is a particularly important attribute for team sport athletes. Furthermore, it has been shown that in athletes who have suffered significant lower limb injures that the ability to produce force quickly is often attenuated in both the acute rehab and post return to play period $(37,38)$. Thus, the current results present a useful method of within resistance training session organisation that can augment RSI for concurrently trained athletes such as team sport athletes.

The relationship between PAPE and fatigue is important when optimising the potentiation response. AF and more broadly team sport athletes possess a high aerobic capacity, and as team sports require repeated high-intensity actions throughout training and matches this may explain the present results showing the ability to recover adequately when utilising short duration 
305

306

307

308

309

310

311

312

313

314

315

316

317

318

319

320

321

322

323

324

325

326

327

recovery periods. The present results show no detrimental effect of short duration $(<4 \mathrm{~min})$

recovery periods on subsequent kinetic or kinematic variables of jump performance and that 1-

min was more beneficial than 4-min. Previous research utilising 11 university rugby union

players tested the effect of a 5-RM back squat on PAPE and subsequent CMJ performance with results showing a 2.9\% increase in CMJ height 4 minutes after a 5-RM back squat (17). This

finding may be explained by research showing twitch potentiation to be greatest immediately

following a prior conditioning contraction $(9,39)$. However, although twitch potentiation may be

the greatest immediately following a prior conditioning contraction, there also exists a high level

of fatigue that could limit subsequent maximal performance $(4,40)$. For example, Pearson and

Hussain (40) observed an increase in twitch torque of the knee extensors but no significant effect on CMJ jump height, jump power, rate of force development or take-off velocity 4 minutes post either a 3, 5 or 7 second isometric half squat MVC in recreationally trained men. The researchers suggested that PAPE was repressed by fatigue in the other musculature used during the conditioning stimulus, where twitch torque was only measured in the quadriceps (40). This may suggest a reciprocal relationship between fatigue and potentiation whereby if fatigue is favoured then a decrease in performance can be expected. Consequently, if potentiation is more pronounced, then an increase in performance might be expected (41).

In the present study, the participants preceded the resistance training session by a sport-specific endurance training session typically completed as part of their normal training routines. The prior training suggests some residual fatigue upon commencing the subsequent resistance training session undertaken one hour later. As such, participants were instructed to self-select their conditioning stimulus load while being told to lift as heavy as they could do safely. Participant's 
328 self-selected conditioning stimulus equated to $74 \% \pm 9 \%$ of $1-\mathrm{RM}$ which is less than the

329 previous PAPE recommendations of 80\% 1-RM (10). The acute strength 'state' of concurrent

330 training athletes undertaking preceding high-intensity intermittent activities can fluctuate based

331 on arousal, preceding activity, diet and sleep (42) inferring that the preceding sport-specific

332 endurance session may influence subsequent resistance training performance. Consistent with

333 this notion, it has been previously demonstrated that sport-specific endurance running loads

334 negatively affect subsequent same day RT performance (23), suggesting that team sport athletes

335 may not lift $>80 \%$ 1-RM due to residual fatigue. In support of the efficacy of lower conditioning

336 stimulus loads, previous research in national level Olympic lifters report increased CMJ

337 performance following 'moderate' loads (45-75\% RM) in a back squat (43). In addition, a meta-

338 analysis indicates that moderate (60-84\%) vs heavy loads (>85\%) (ES: 1.06 vs 0.31), multiple set

339 vs single set (ES: 0.66 vs 0.24 ) and athletic vs untrained cohorts (ES: 0.81 vs 0.14 ) result in an

340 increase in power augmentation in subsequent potentiation tasks (44). Greater RT experience

341 results in increased neural firing, motor unit synchronisation (45) and elevated myosin regulatory

342 light chain phosphorylation activity $(13,15,16)$. As such, it may be suggested that greater

343 training experience facilitates the relationship between potentiation and fatigue, and the acute

344 strength 'state' of the athlete can fluctuate, thus, current recommendations of $>80 \% 1-\mathrm{RM}$ for the

345 conditioning contraction stimulus should only be considered a guideline.

347 Contrast training offers another method of within resistance training periodisation. However,

348 little research has employed concurrently trained athletes as the experimental cohort $(4,6)$ and

349 no research has assessed the PAPE response in a same day concurrent training model where the

350 preceding field-based skills/endurance training load has been reported. The strength of the 
351 present study was that the preceding skills/endurance training load was matched between the 1-

352 min and 4-min groups. Furthermore, the self-selected conditioning stimulus load was consistent

353 between groups. In addition, the current collection period was undertaken during the in-season

354 period where the focus is solely on being in peak condition for competition and subsequent

355 recovery for the following game. This provides a high level of ecological validity as high

356 performance athletes such as those involved with the present study will often self-select their

357 individual resistance training loads based on their individual recovery status following the most

358 recent competitive match. This meant that athletes lifted under the recommended $80 \% 1-\mathrm{RM}$

359 previously reported in previous PAPE research, making comparisons with research utilising

$360>90 \%$ 1-RM difficult (46-48). Given this 'lower' conditioning stimulus load, it could be

361 suggested that subsequent drop jump performance was compromised. However, drop jump

362 performance following the conditioning stimulus was comparable to baseline meaning there was

363 minimal detrimental effect of the lower prior PAPE stimulus.

364

Despite these novel findings, there are some inherent limitations that should be addressed. The current research utilised ten professional team sport athletes (Australian Rules Footballers) and while this was sufficient for the current analysis, larger sample sizes of athletes from Australian

Rules Football and other team sports may increase the generalisability of these results.

Furthermore, the absence of an 8-12 minute recovery group for comparison as with current contrast training recommendations is a limitation. However, this is not feasible within the constraints of training design within the context of professional team sport athletes. In addition, table 1 shows the ICC and CV for baseline drop jumps, while the ICC reported are classified as

373 good reliability (49), the observed CV are above the desired 5\%. The higher than recommended 
374 CVs reflected the accumulated fatigue on the prior on field session, the relative complex nature

375 of drop jumps compared to squat or countermovement jumps and the fact that a number of the

376 variables e.g. RSI and impulse were calculated using DJ kinetic and temporal measures.

377 However, the ecological validity of testing professional athletes in the field is a strength of the

378 current study design.

The present findings suggest the utilization of PAPE within a same day concurrent training team sport model, where short duration recovery periods between conditioning stimulus and subsequent plyometric (jumping) tasks can be implemented without concern of negatively

384 affecting the subsequent plyometric task performance. If the rest periods required for 385 potentiation to exceed fatigue are too large, it is likely that strength and conditioning coaches working in high performance sporting organisations would have to reduce training volume or increase the duration of each resistance training session, both of which are not practical. As such, PAPE that utilizes short recovery durations provides another within resistance training periodization strategy for team sport athletes. 
390

391

392

393

394

395

396

397

398

399

400

401

402

403

404

405

406

407

408

409

410

411

412

413

414

415

416

417

418

419

420

421

422

423

424

425

426

427

428

429

430

431

432

433

1. Kraemer, WJ, K Adams, E Cafarelli, GA Dudley, C Dooly, MS Feigenbaum, SJ Fleck, B Franklin, AC Fry, JR Hoffman, RU Newton, J Potteiger, MH Stone, NA Ratamess, and T Triplett-McBride. American College of Sports Medicine position stand. Progression models in resistance training for healthy adults. Med Sci Sports Exerc 34: 364-380, 2002.

2. Kraemer , W, K Adams, E Cafarelli, G Dudley, C Dooly, M Feigenbaum, S Fleck, B Franklin, A Fry, J Hoffman, R Newton, J Potteiger, M Stone, N Ratamess, and T Triplett-McBride. American College of Sports Medicine position stand. Progression models in resistance training for healthy adults. Med Sci Sports Exerc 41: 687-708, 2009.

3. Maio Alves, JM, AN Rebelo, C Abrantes, and J Sampaio. Short-term effects of complex and contrast training in soccer players' vertical jump, sprint, and agility abilities. J Strength Cond Res 24: 936-941, 2010.

4. Comyns, TM, AJ Harrison, L Hennessy, and RL Jensen. Identifying the optimal resistive load for complex training in male rugby players. Sports Biomech 6: 59-70, 2007.

5. Argus, CK, ND Gill, JW Keogh, MR McGuigan, and WG Hopkins. Effects of two contrast training programs on jump performance in rugby union players during a competition phase. Int J Sports Physiol Perform 7: 68-75, 2012.

6. Mola, JN, SS Bruce-Low, and SJ Burnet. Optimal recovery time for postactivation potentiation in professional soccer players. J Strength Cond Res 28: 1529-1537, 2014.

7. Struzik, A, G Juras, B Pietraszewski, and A Rokita. Effect of drop jump technique on the reactive strength index. Journal of human kinetics 52: 157-164, 2016.

8. Gossen, ER and DG Sale. Effect of postactivation potentiation on dynamic knee extension performance. Eur J Appl Physiol 83: 524-530, 2000.

9. Hamada, T, DG Sale, JD MacDougall, and MA Tarnopolsky. Postactivation potentiation, fiber type, and twitch contraction time in human knee extensor muscles. J Appl Physiol (1985) 88: 2131-2137, 2000.

10. Tillin, NA and D Bishop. Factors modulating post-activation potentiation and its effect on performance of subsequent explosive activities. Sports Med 39: 147-166, 2009.

11. Blazevich, AJ and N Babault. Post-activation Potentiation Versus Post-activation Performance Enhancement in Humans: Historical Perspective, Underlying Mechanisms, and Current Issues. 10, 2019.

12. Gouvêa, AL, IA Fernandes, EP César, WAB Silva, and PSC Gomes. The effects of rest intervals on jumping performance: A meta-analysis on post-activation potentiation studies. Journal of Sports Sciences 31: 459-467, 2013.

13. Sweeney, HL and JT Stull. Alteration of cross-bridge kinetics by myosin light chain phosphorylation in rabbit skeletal muscle: implications for regulation of actin-myosin interaction. Proceedings of the National Academy of Sciences of the United States of America 87: 414-418, 1990.

14. Szczesna, D. Regulatory light chains of striated muscle myosin. Structure, function and malfunction. Curr Drug Targets Cardiovasc Haematol Disord 3: 187-197, 2003.

15. Hodgson, M, D Docherty, and D Robbins. Post-activation potentiation: underlying physiology and implications for motor performance. Sports Med 35: 585-595, 2005. 
434

435

436

437

438

439

440

441

442

443

444

445

446

447

448

449

450

451

452

453

454

455

456

457

458

459

460

461

462

463

464

465

466

467

468

469

470

471

472

473

474

475

476

477

478

479

480
16. Baudry, S and J Duchateau. Postactivation potentiation in a human muscle: effect on the loadvelocity relation of tetanic and voluntary shortening contractions. J Appl Physiol (1985) 103: 1318-1325, 2007.

17. Mitchell, CJ and DG Sale. Enhancement of jump performance after a 5-RM squat is associated with postactivation potentiation. Eur J Appl Physiol 111: 1957-1963, 2011.

18. Tillin, NA and D Bishop. Factors modulating post-activation potentiation and its effect on performance of subsequent explosive activities. Sports medicine 39: 147-166, 2009.

19. Gamble, P. Periodization training for team sport athletes. Journal of Strength and Conditioning vol. 28: pp. 56-66, 2006.

20. Ritchie, D, WG Hopkins, M Buchheit, J Cordy, and JD Bartlett. Quantification of training and competition load across a season in an elite Australian football club. International Journal of Sports Physiology and Performance 11: 474-479, 2016.

21. Bilsborough, JC, KG Greenway, DA Opar, SG Livingstone, JT Cordy, SR Bird, and AJ Coutts. Comparison of anthropometry, upper-body strength, and lower-body power characteristics in different levels of Australian football players. J Strength Cond Res 29: 826-834, 2015.

22. Juhari, F, D Ritchie, F O'Connor, N Pitchford, M Weston, HR Thornton, and JD Bartlett. The Quantification of Within-Week Session Intensity, Duration, and Intensity Distribution Across a Season in Australian Football Using the Session Rating of Perceived Exertion Method. Int J Sports Physiol Perform 13: 940-946, 2018.

23. Ritchie, D, J Keogh, S Stern, P Reaburn, F O'Connor, and JD Bartlett. The Effects of EnduranceBased Skills-Specific Running Loads on Same-Day Resistance-Training Performance in Professional Australian Rules Football Players. Int J Sports Physiol Perform: 1-8, 2020.

24. O'Connor, F, H Thornton, D Ritchie, J Anderson, L Bull, A Rigby, Z Leonard, S Stern, and J Bartlett. Greater association of relative thresholds than absolute thresholds with noncontact lower-body injury in professional Australian rules footballers: Implications for sprint monitoring. International Journal of Sports Physiology and Performance: 1-25, 2019.

25. Rampinini, E, A Bosio, I Ferraresi, A Petruolo, A Morelli, and A Sassi. Match-related fatigue in soccer players. Medicine \& Science in Sports \& Exercise 43: 2161-2170, 2011.

26. Jennings, D, S Cormack, AJ Coutts, L Boyd, and RJ Aughey. The validity and reliability of GPS units for measuring distance in team sport specific running patterns. International Journal of Sports Physiology and Performance 5: 328-341, 2010.

27. Bosco, C, P Luhtanen, and PV Komi. A simple method for measurement of mechanical power in jumping. Eur J Appl Physiol Occup Physiol 50: 273-282, 1983.

28. Young, WB, GJ Wilson, and C Byrne. A comparison of drop jump training methods: effects on leg extensor strength qualities and jumping performance. Int J Sports Med 20: 295-303, 1999.

29. Markovic, G, D Dizdar, I Jukic, and M Cardinale. Reliability and factorial validity of squat and countermovement jump tests. J Strength Cond Res 18: 551-555, 2004.

30. McGuigan, MR, TL Doyle, M Newton, DJ Edwards, S Nimphius, and RU Newton. Eccentric utilization ratio: effect of sport and phase of training. J Strength Cond Res 20: 992-995, 2006.

31. Cormack, SJ, RU Newton, MR McGuigan, and TL Doyle. Reliability of measures obtained during single and repeated countermovement jumps. Int J Sports Physiol Perform 3: 131-144, 2008.

32. Buckthorpe, M, J Morris, and JP Folland. Validity of vertical jump measurement devices. J Sports Sci 30: 63-69, 2012.

33. Cohen, J. Statistical Power Analysis for the Behavioral Sciences (2nd ed.). 1988, Hillsdale, NJ: Lawrence Erlbaum Associates, Publishers.

34. French, DN, WJ Kraemer, and CB Cooke. Changes in dynamic exercise performance following a sequence of preconditioning isometric muscle actions. J Strength Cond Res 17: 678-685, 2003. 
481

482

483

484

485

486

487

488

489

490

491

492

493

494

495

496

497

498

499

500

501

502

503

504

505

506

507

508

509

510

511

512

513

514

515

516

517

518

519

520

521

522

523

35. Schmidtbleicher, D, Neurophysiologische Aspekte des Sprungkrafttrainings (Neurophysiological aspects of jump training), K. Carl and J. Schiffer, Editors. 1986, Bundesinstitut fur Sportwissenschaft: Koln. p. 56-72.

36. Ebben, $\mathrm{W}$ and $\mathrm{E}$ Petushek. Using the Reactive Strength Index Modified to Evaluate Plyometric Performance. Journal of strength and conditioning research / National Strength \& Conditioning Association 24: 1983-1987, 2010.

37. Read, PJ, S Michael Auliffe, MG Wilson, and P Graham-Smith. Lower Limb Kinetic Asymmetries in Professional Soccer Players With and Without Anterior Cruciate Ligament Reconstruction: Nine Months Is Not Enough Time to Restore "Functional" Symmetry or Return to Performance. Am J Sports Med 48: 1365-1373, 2020.

38. Jordan, MJ, N Morris, M Lane, J Barnert, K MacGregor, M Heard, S Robinson, and W Herzog. Monitoring the Return to Sport Transition After ACL Injury: An Alpine Ski Racing Case Study. 2, 2020.

39. Folland, JP, T Wakamatsu, and MS Fimland. The influence of maximal isometric activity on twitch and $\mathrm{H}$-reflex potentiation, and quadriceps femoris performance. Eur J Appl Physiol 104: 739-748, 2008.

40. Pearson, SJ and SR Hussain. Lack of association between postactivation potentiation and subsequent jump performance. European Journal of Sport Science 14: 418-425, 2014.

41. Rassier, DE and BR Macintosh. Coexistence of potentiation and fatigue in skeletal muscle. Braz J Med Biol Res 33: 499-508, 2000.

42. McBurnie, A, K Allen, G Maybanks, M McDwyer, T Dos'Santos, P Jones, P Comfort, and J McMahon. The Benefits and Limitations of Predicting One Repetition Maximum Using the LoadVelocity Relationship. Strength and Conditioning Journal 41: 1, 2019.

43. Fukutani, A, S Takei, K Hirata, N Miyamoto, H Kanehisa, and Y Kawakami. Influence of the intensity of squat exercises on the subsequent jump performance. J Strength Cond Res 28: 22362243, 2014.

44. Wilson, JM, NM Duncan, PJ Marin, LE Brown, JP Loenneke, SM Wilson, E Jo, RP Lowery, and C Ugrinowitsch. Meta-analysis of postactivation potentiation and power: effects of conditioning activity, volume, gender, rest periods, and training status. J Strength Cond Res 27: 854-859, 2013.

45. Folland, JP and AG Williams. The adaptations to strength training : morphological and neurological contributions to increased strength. Sports Med 37: 145-168, 2007.

46. Kilduff, LP, N Owen, H Bevan, M Bennett, MIC Kingsley, and D Cunningham. Influence of recovery time on post-activation potentiation in professional rugby players. Journal of Sports Sciences 26: 795-802, 2008.

47. Young, WB, A Jenner, and K Griffiths. Acute Enhancement of Power Performance From Heavy Load Squats. The Journal of Strength \& Conditioning Research 12: 82-84, 1998.

48. Duthie, GM, WB Young, and DA Aitken. The acute effects of heavy loads on jump squat performance: an evaluation of the complex and contrast methods of power development. $J$ Strength Cond Res 16: 530-538, 2002.

49. Koo, TK and MY Li. A Guideline of Selecting and Reporting Intraclass Correlation Coefficients for Reliability Research. Journal of chiropractic medicine 15: 155-163, 2016. 


\section{Figure 1}

Timeline shows team-based skills/endurance session in the morning followed by resistance training in the afternoon with either 1-min or 4-min recovery prescribed within contrast training on separate training days.

Contrast
\begin{tabular}{|l|} 
Skills = Field based skills/Endurance \\
Cond = Additional Running \\
RES = Squat/ Bench Press/Bench \\
Pull + Supplementary exercises \\
S = Strength \\
P = Plyometric \\
Hyp = Hypertrophy
\end{tabular}

\section{Skills} Cond
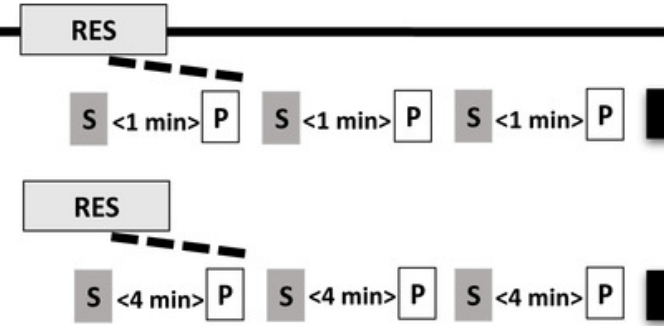
Figure 2

A box and whisker plot showing baseline drop jump performance for 1-min and 4-min protocols. The box represents $25^{\text {th }}$ and $75^{\text {th }}$ percentiles and the bars represent minimum and maximum values. 
a

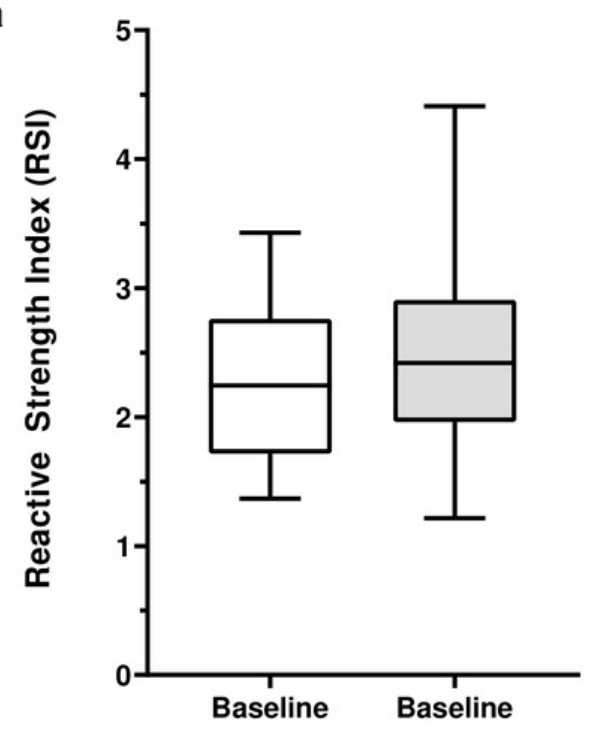

C

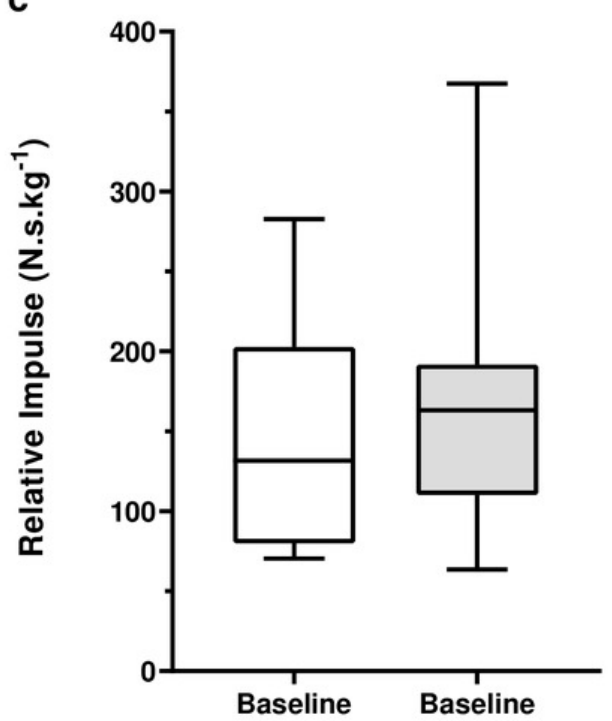

e

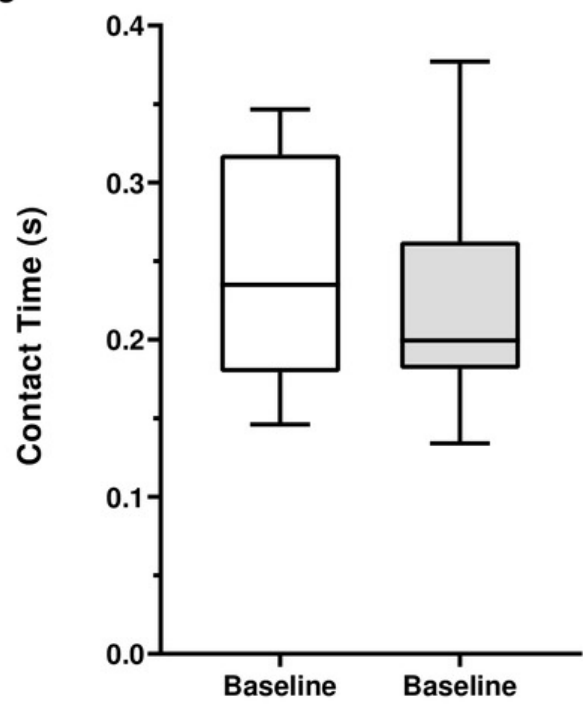

b

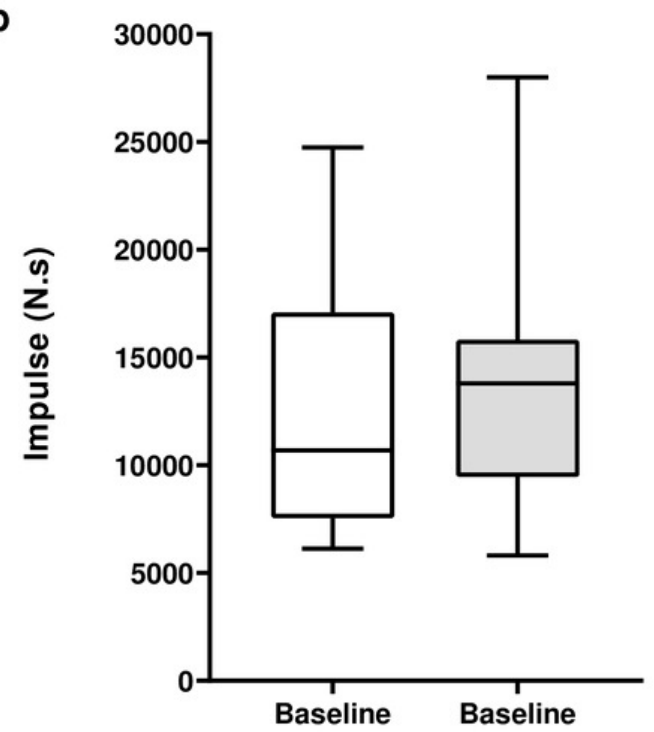

d

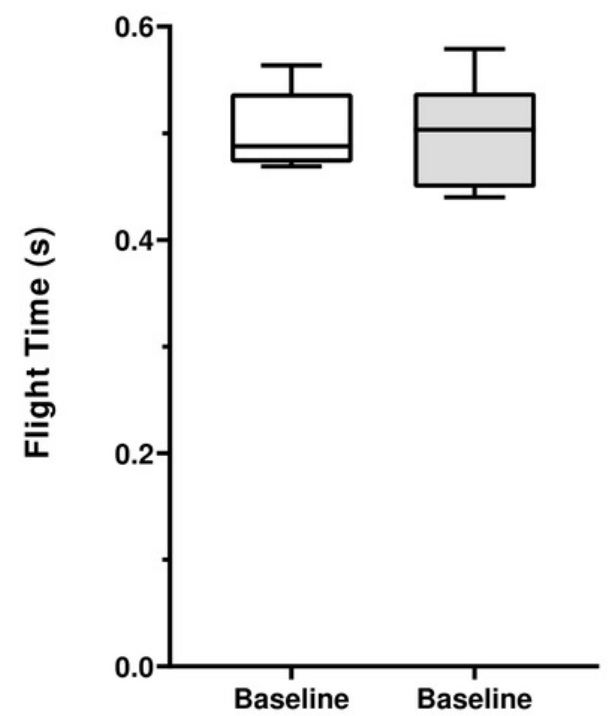


Figure 3

Baseline and set comparisons for 1-min protocols. Bars represent mean and SD values, with individual data points plotted. * indicates $\mathrm{P}$ value $<0.05$. 
a

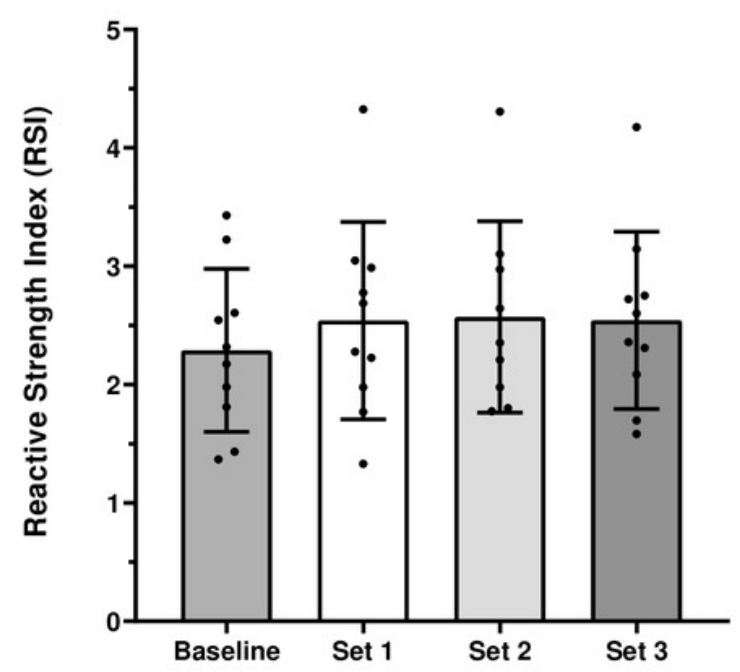

C

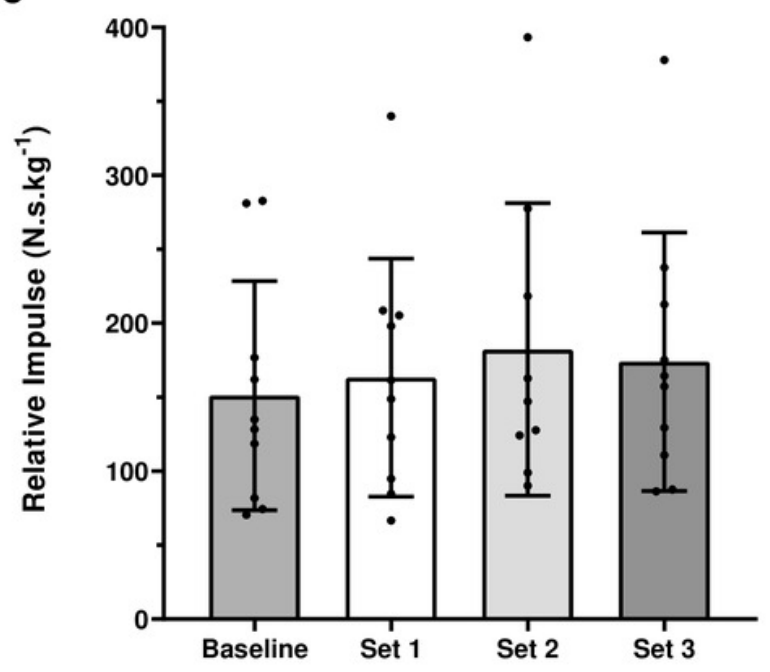

b

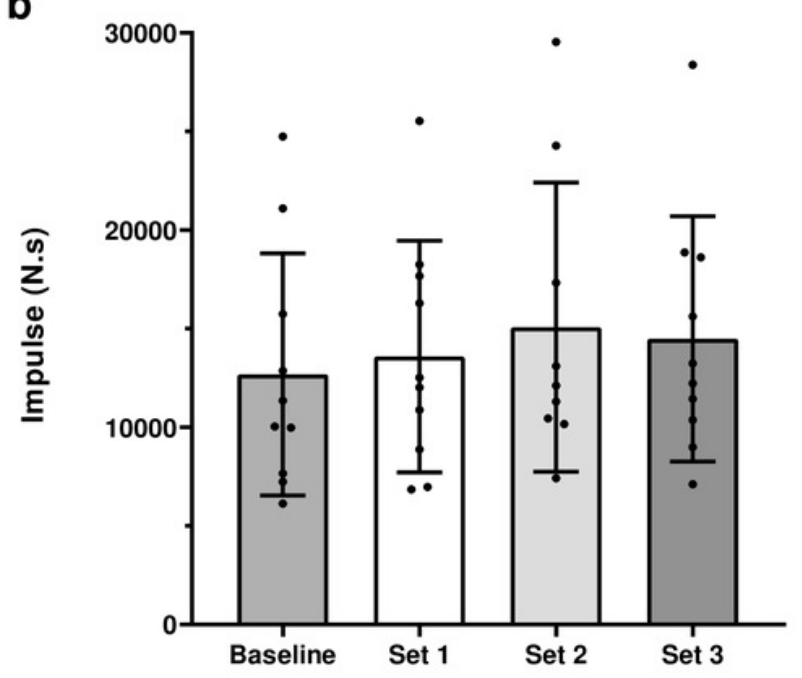

d

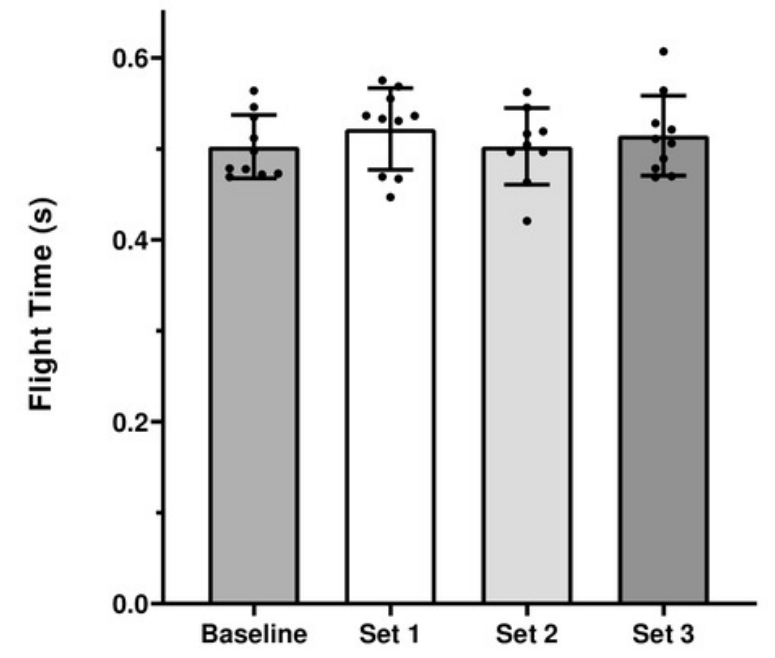

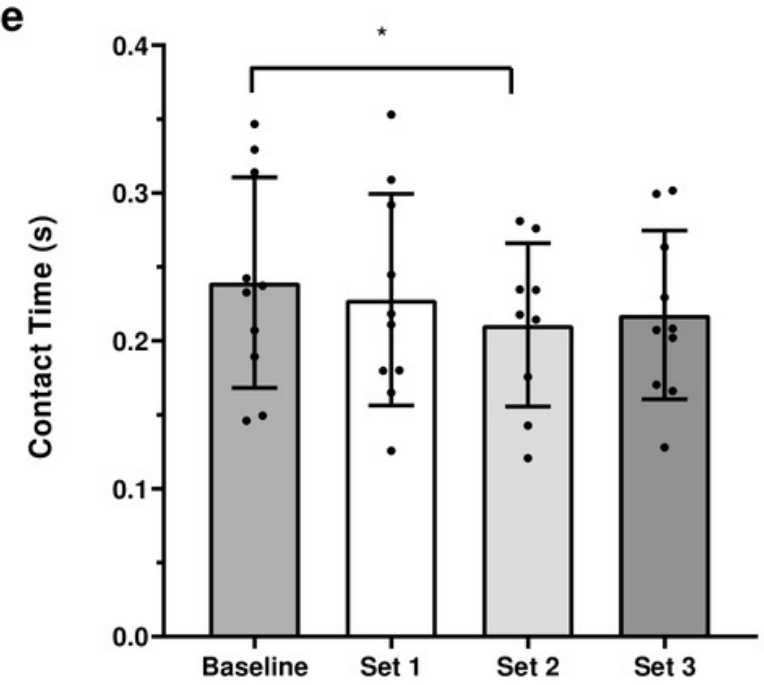


Figure 4

Baseline and set comparisons for 4-min protocols. Bars represent mean and SD values, with individual data points plotted. * indicates $\mathrm{P}$ value $<0.05$. 
a

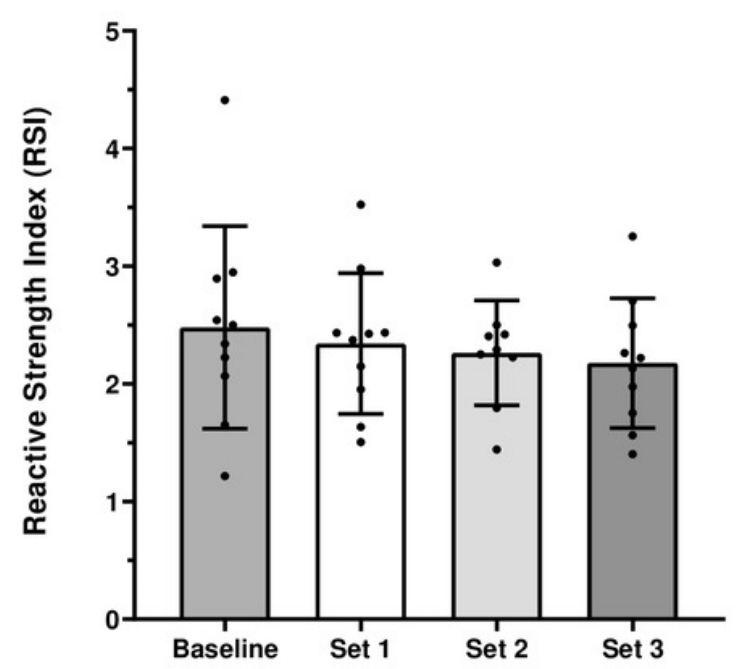

C

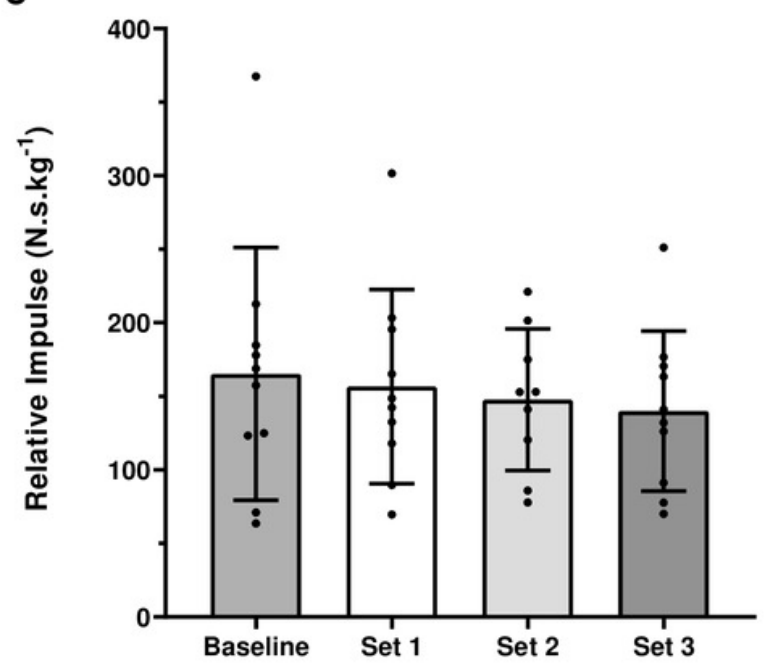

e

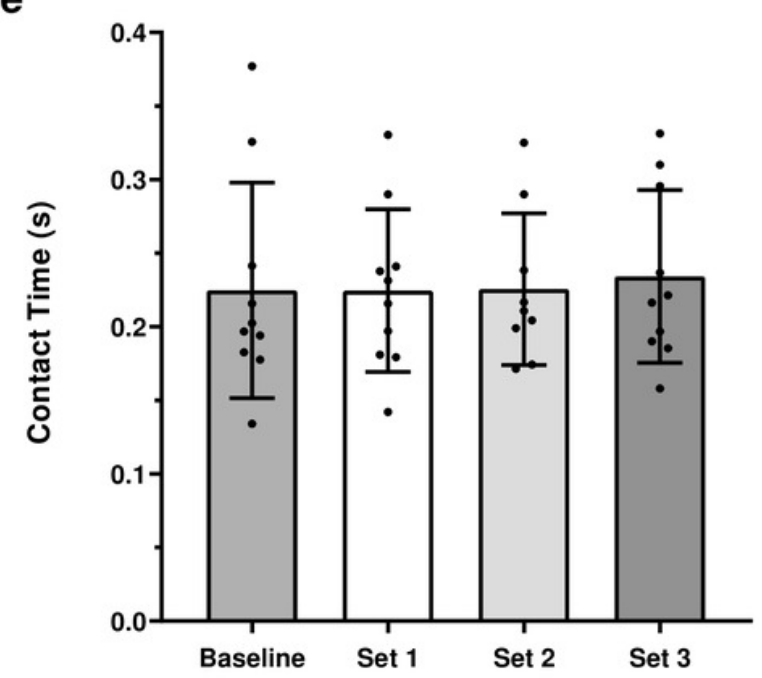

b

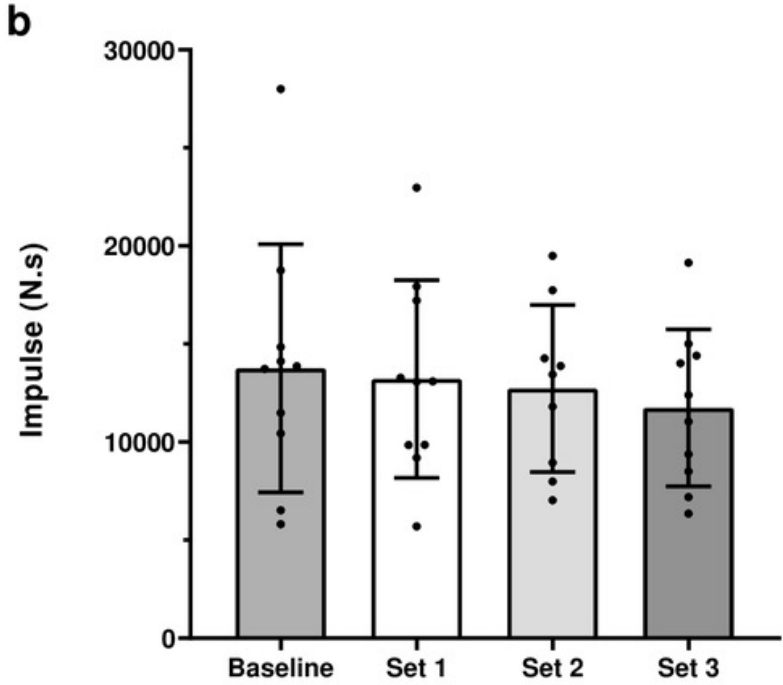

d

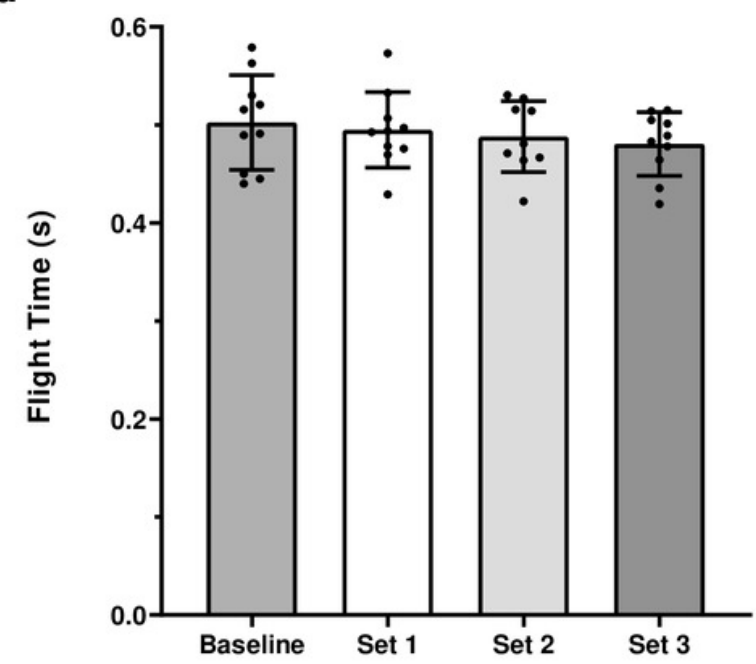


Figure 5

Between condition set comparisons for 1-min and 4-min protocols. Bars represent mean and SD values, with individual data points plotted. $*$ indicates $P$ value $<0.05$. 
a

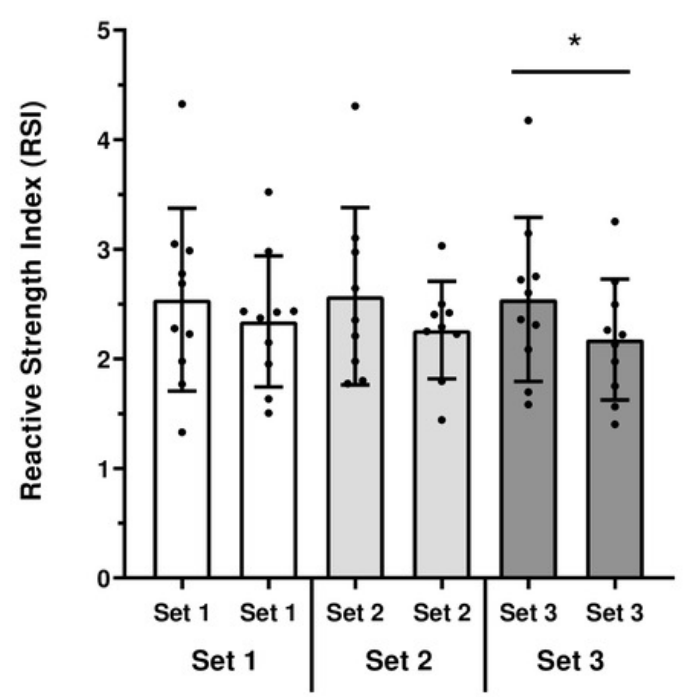

C

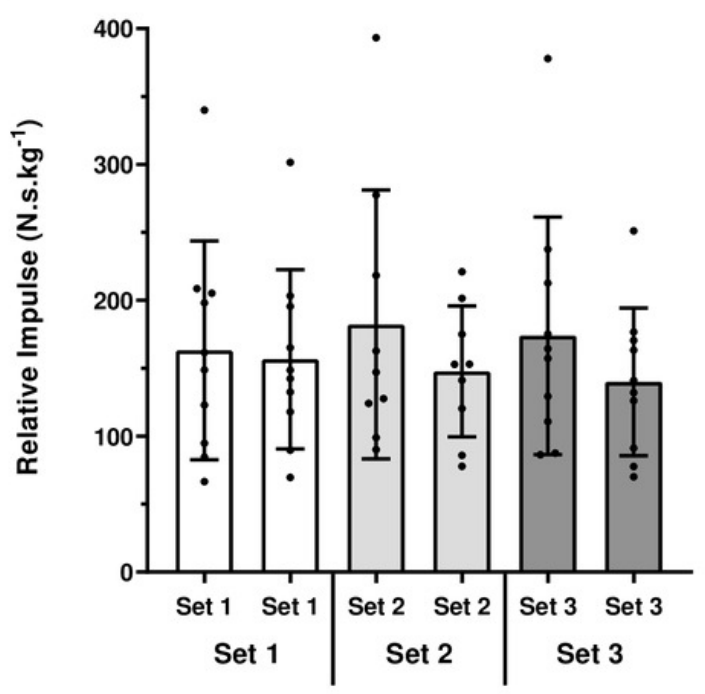

b

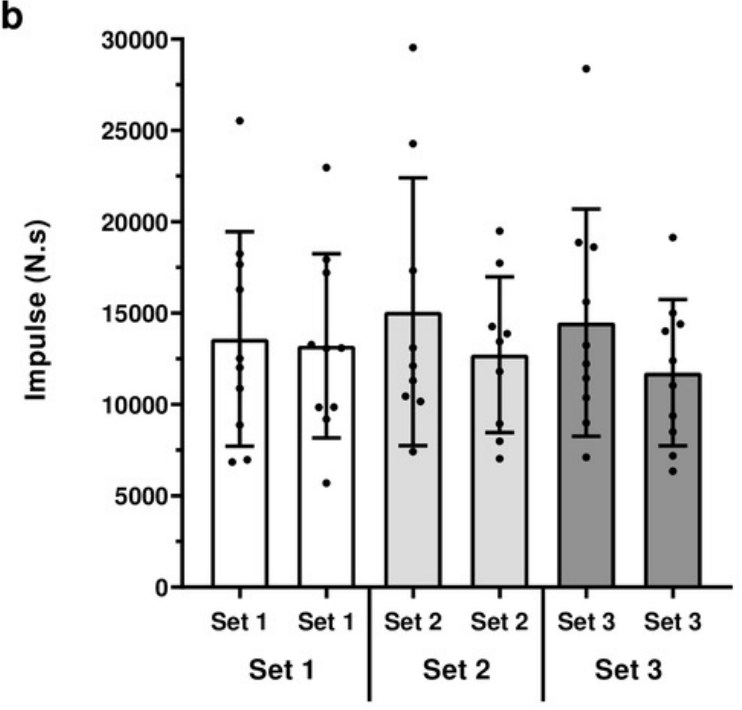

d

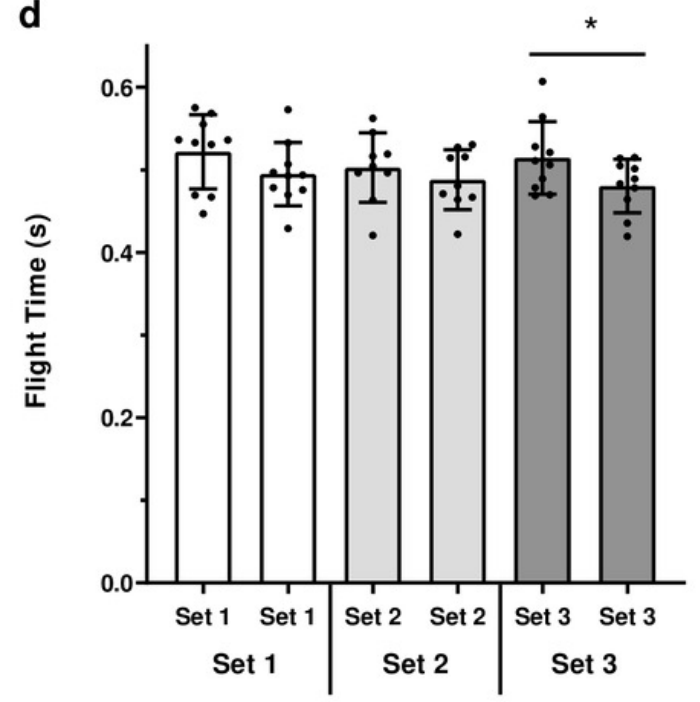

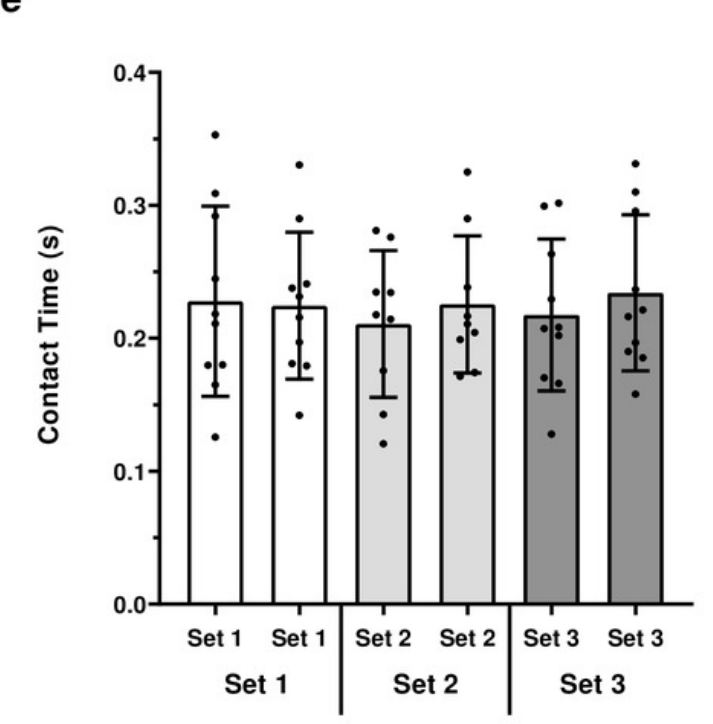




\section{Table 1 (on next page)}

Intraclass Correlation (ICC) and Coefficient of Variation (CV) for baseline drop jumps. 
Table 1. Intraclass Correlation (ICC) and Coefficient of Variation (CV) for baseline drop jumps.

1

2

\begin{tabular}{lcc} 
RSI (reactive strength index) & 0.85 & 14.5 \\
Impulse (N.s) & 0.83 & 23.0 \\
Relative Impulse (N.s.kg-1) & 0.86 & 23.0 \\
Flight Time (FT) (s) & 0.54 & 7.1 \\
Contact Time (CT) (s) & 0.86 & 12.9 \\
\hline
\end{tabular}




\section{Table 2 (on next page)}

Comparisons of 1 min vs 4 min protocols for preceding sports specific running loads. 
Table 2. Comparisons of $1 \mathrm{~min}$ vs $4 \mathrm{~min}$ protocols for preceding sports specific running loads.

Total Distance (m)

High Speed Running $(>15 \mathrm{~km} / \mathrm{h})$

Distance covered $>75 \%(\mathrm{~m}) \quad$ Distance covered $>85 \%(\mathrm{~m})$

$\mathbf{M} / \mathbf{m i n}$

$1 \mathrm{~min}$

$4 \min$

$1 \min$

$4 \mathrm{~min}$

$1 \mathrm{~min}$

$4 \min$

$1 \mathrm{~min}$

$4 \min$

1 min

4 min

Mean (SD)

7912 (1258)

$2338(772)$

$2280(829)$

$138(80.4)$

159 (113)

34.5 (26.7)

$42.9(45.2)$

$99(13.7)$

$\mathrm{P}$ value

0.724

0.800

0.695

0.882

0.695

ES $(95 \% \mathrm{CI})$

$0.08(-0.80-0.96)$

$0.07(-0.81-0.95)$

$-0.21(-1.08-0.67)$

$-0.22(-1.10-0.66)$

$0.19(-0.68-1.07)$

1 


\section{Table 3 (on next page)}

Comparisons of $1 \mathrm{~min}$ vs 4 min protocols for total tonnage of preceding conditioning stimulus (box squat). 
Table 3. Comparisons of 1 min vs 4 min protocols for total tonnage of preceding conditioning stimulus (box squat).

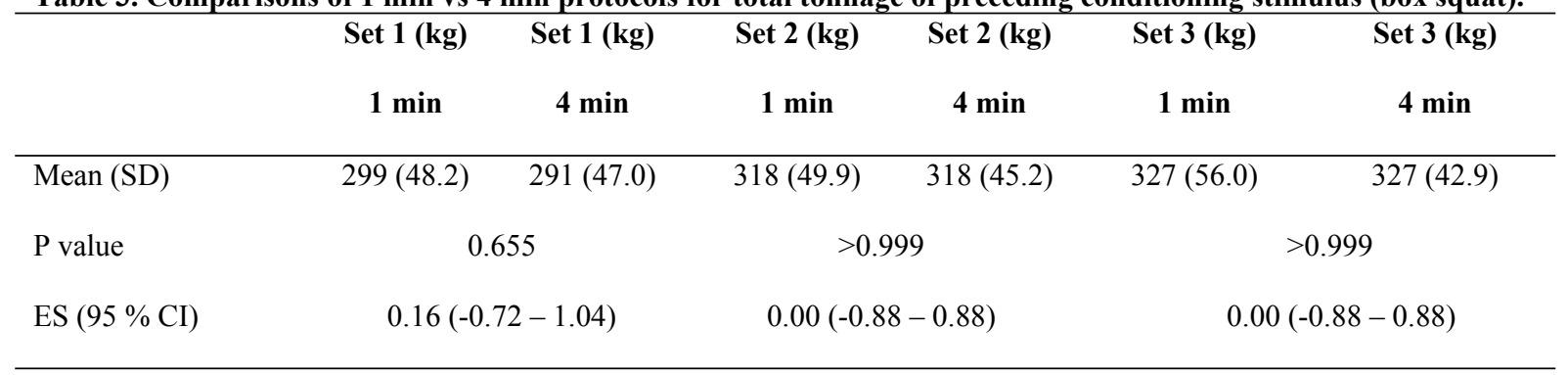

